\title{
Versión mexicana de la Escala de uso del tiempo libre para el afrontamiento (LCSS-M)
}

\section{Mexican version of the Leisure coping strategy scale (LCSS-M)}

\author{
Minerva Vanegas Farfano ${ }^{1} \quad$ Mónica González Ramírez \\ Universidad Autónoma de Nuevo León \\ (Rec: marzo 2014 - Acep: junio 2014)
}

\begin{abstract}
Resumen
El presente estudio analiza las propiedades psicométricas del cuestionario Leisure Coping Strategy Scale (LCSS) en población mexicana, nombrada como LCSS-M. Método: La escala se analizó con una muestra de 141 adultos. Su validez convergente fue obtenida mediante la Escala de Estrés Percibido (PSS) y el Inventario de Satisfacción con la Vida. La estructura factorial de las tres subescalas se revisó mediante un análisis factorial exploratorio con el método de componentes principales y rotación VARIMAX; posteriormente se hizo el análisis factorial confirmatorio. Resultados: Ambas escalas, de estrés percibido y satisfacción con la vida, aportaron evidencia de la validez convergente en ítems. Los análisis factoriales, exploratorio y confirmatorio, apoyan la dimensionalidad y estructura en los tres factores del modelo original. La consistencia interna del inventario refleja una adecuada confiabilidad. En general éste estudio indica a la LCSS-M como una medida valida y fiable para el estudio del uso de pasatiempos como estrategia de afrontamiento en población mexicana.
\end{abstract}

Palabras clave: afrontamiento, estrés, pasatiempo y salud.

\begin{abstract}
Objective: The present study tested the psychometric properties of the Leisure Coping Strategy Scale (LCSS), named as LCSS-M, in Mexican population. Method: Survey in a sample of 141 adults. Evidence of convergent validity was obtained with the PSS and Satisfaction with Life Scale. The factor structure of the three subscales were examined through an exploratory factor analysis using the principal component method with VARIMAX rotation, subsequently confirmatory factor analysis was performed.

Results: Both scales, PSS and Satisfaction with Life, gave evidence of convergent validity. Also, exploratory and confirmatory factor analysis, support the dimensionality and factor structure of the original three factor model. The internal consistencies of the subscales were acceptable. The present study shows that the LCSS-M seems to be a valid and reliable measure for Mexican samples for future research on leisure as a coping strategy.
\end{abstract}

Keywords: coping, stress, leisure, leisure and health.

\footnotetext{
Correspondencia dirigida a: Minerva Vanegas Farfano, Universidad Autónoma de Nuevo León, UANL, Fac. de Psicología. Av. Universidad S/N Ciudad Universitaria San Nicolás de los Garza Nuevo León, C.P. 66451 México. Teléfono: 83294050. Correo electrónico: minerva.vanegas@gmail.com.
} 


\section{La salud y lo cotidiano}

El desarrollo de investigaciones que ligan a la salud psicológica y el ajuste al ambiente no es nuevo, lo que no implica que no se hayan generado cambios en el tratamiento de sus temáticas; pues se encuentra en constante desarrollo. A la par del uso de métodos mixtos para ahondar en el estudio de estrategias de afrontamiento no previstas en los cuestionarios (Cano, Rodríguez \& García, 2007); otros grupos y espacios de conocimiento están siendo incluidos (Mactavish \& Iwasaki, 2005). Gracias a ello, los vínculos entre las diversas actividades que realizan las personas en lo cotidiano y el impacto en su salud se conocen. Este documento presenta la adaptación de un inventario enfocado al uso del tiempo libre y los pasatiempos como una estrategia de afrontamiento, actividad cuyo impacto en la salud se explora poco en México.

\section{Estudio del estrés y la salud psicológica en poblaciones de habla hispana}

A raíz de su estudio, hoy se sabe que el estrés genera respuestas fisiológicas esenciales para la supervivencia. Si bien las demandas de la vida son distintas en cada época, actualmente el estrés psicológico perdura y se vincula a problemas en la salud mental y física (Mactavish \& Iwasaki, 2005; Mattingly \& Sayer, 2006). El estrés, explicado como el resultado del desbalance entre las demandas del ambiente y los recursos disponibles de la persona para responder a estos, conlleva el desarrollo de conductas para afrontarle (Miller \& McCool, 2003).

Existen diversos modelos teóricos sobre el estrés. Desde la teoría transaccional (Lazarus \& Folkman, 1987) el estudio del afrontamiento se centra en la intensidad de los conflictos y las diferentes estrategias manejadas (Miller \& McCool, 2003). El afrontamiento permite la modulación o adaptación ante eventos estresantes (Morán, Landero \& González, 2010); a pesar de que éste obedece a una naturaleza multidimensional en donde se utilizan estrategias, tanto cognitivas como conductuales para manejarle, se trata de una actividad específica realizada por la persona ante el estresor (Iwasaki \& Mannell, 2000; Mactavish \& Iwasaki, 2005). Debido a la posibilidad para reconocer diferencias individuales al valorar resultados, aquellos modelos enfocados en la evaluación individual de las amenazas y los recursos percibidos son los más utilizados (Hamarat et al., 2001).
Actualmente las investigaciones en poblaciones de habla hispana en torno al tópico se concentran en el manejo del estrés en muestras con alguna enfermedad o relacionados a una dolencia (Paz-Rodríguez \& Vázquez-Velez, 2012; Peralta-Ramírez, Robles-Ortega, Navarrete-Navarrete \& Jiménez-Alonso, 2009), se enfocan en el estrés o estrés y burnout en ambientes académicos o laborales (Hernández, Olmedo \& Ibañez, 2003) u observan su relación con otros conceptos como el bienestar subjetivo en el ámbito laboral (Hernández et al., 2003; Paris \& Omar, 2009). El desarrollo de escalas o sus adaptaciones se encuentran estrechamente vinculados a estos temas (Nava, Ollua, Vega \& Soria, 2010; Olmedo, Ibáñez \& Hernández, 2001). Entre los inventarios encontrados en recientes publicaciones mexicanas, se citan la adaptación española del Inventario de Estrategias de Afrontamiento (Cano et al., 2007), la Escala de modos de Afrontamiento (Nava-Quiroz, Vega-Valero \& Soria, 2010) y la adaptación del Brief COPE o COPE-28 (Morán et al., 2010). Si bien estos varían en la cantidad y tipo de estrategias utilizadas como forma de afrontamiento, todas ellas se vinculan con la teoría transaccional (Lazarus \& Folkman, 1987) ya mencionada.

Recientemente, los estudios sobre los pasatiempos en países como Canadá y Australia, han mostrado que las actividades durante el tiempo libre permiten resultados positivos para la persona al identificarlos como una forma de mantenimiento o mejora de la salud. De acuerdo a éstas investigaciones, tanto cualitativas como cuantitativas, estos presentan la capacidad de proporcionar redes de apoyo social, aumentar el estado de ánimo y mejorar la disposición para enfrentar el estrés (Iwasaki \& Mannell, 2000; Mattingly \& Sayer, 2006). Hoy en día los estudios sobre pasatiempos en población de habla hispana exploran los efectos del estrés en deportistas de alto desempeño y factores relacionados (Bueno, Fernández-Castro \& Capdevelia, 2001; Ortin, Olmedilla, Garcés de los Fayos \& Hidalgo, 2008). En otras palabras, si bien existen las investigaciones que señalan un vínculo entre el estrés, sus estrategias de afrontamiento, el bienestar subjetivo y la interrelación entre diversas actividades de la vida cotidiana, el estudio del impacto de los pasatiempos y uso del tiempo libre como estrategia de afrontamiento y el análisis y/o adaptación de pruebas utilizadas con estos fines, no ha sido desarrollado.

Teniendo en cuenta que actualmente se carece en México de estudios enfocados a la investigación de los pasatiempos como una estrategia de afrontamiento ante el estrés, el propósito del presente estudio es analizar 
las propiedades psicométricas del cuestionario Leisure Coping Strategy Scale o Escala de uso del tiempo libre para el afrontamiento (LCSS, por sus siglas en inglés) de Iwasaki \& Mannell (2000) en población mexicana.

\section{Escala del uso de tiempo libre para el afrontamiento}

La escala de uso del tiempo libre para el afrontamiento (LCSS; Iwasaki \& Mannell, 2000) es un inventario desarrollado para evaluar el alcance de los pasatiempos como una forma de afrontamiento al estrés, consiste en tres subescalas (de 6 ítems cada una) que miden conceptualmente distintos aspectos del uso de los pasatiempos como estrategia de afrontamiento. El inventario se realizó en base a la discusión teórica y aspectos empíricos relacionados con las propiedades de los pasatiempos como estrategia de afrontamiento. En su versión original la escala de uso del tiempo libre para el afrontamiento (LCSS; Iwasaki \& Mannell, 2000) fue desarrollada y validad con una muestra de 247 participantes, todos ellos alumnos inscritos en el primer año de una universidad canadiense; en ella los ítems fueron planteados en términos de la evaluación de una situación en específico, mismos que son respondidos utilizando una escala ordinal con alternativas del 1 al 7, entre "Totalmente en desacuerdo" y "Totalmente de acuerdo". Sus creadores concuerdan en que la escala de uso del tiempo libre para el afrontamiento (LCSS; Iwasaki \& Mannell, 2000) es un inventario que puede ser útil para identificar de qué manera son utilizados los pasatiempos en el manejo del estrés con distintos niveles de especificidad, dependiendo de la naturaleza del estudio, tanto en las áreas de salud como pasatiempos y manejo de tiempo libre (Iwasaki \& Mannell, 2000). La escala de uso del tiempo libre para el afrontamiento (LCSS; Iwasaki \& Mannell, 2000) incluye tres subescalas:

1. "Compañerismo en el tiempo libre/pasatiempo" que revisa la presencia de experiencias agradables compartidas con los compañeros de actividad consideradas como formas de apoyo social (6 ítems).

2. "Afrontamiento paliativo y el tiempo libre/pasatiempo" que evalúa el reconocimiento del pasatiempo (cognición y acto) como una estrategia para afrontar el estrés (6 ítems).

3. "Mejoría del humor mediante el pasatiempo" la cual incluye seis ítems enfocados al reconocimiento del pasatiempo como un medio que favorece el aumento del estado de ánimo positivo o la reducción del estado de ánimo negativo.

\section{Método}

\section{Participantes}

Siguiendo la regla general de un mínimo de cinco observaciones por variable analizada, se estimó una muestra mínima de 90 participantes (Carretero-Dios \& Pérez, 2005; Hair, Anderson, Tatham \& Black, 1998). Se realizó un muestreo no probabilístico por conveniencia (Meltzoff, 2011) en personas inscritas en los talleres de un museo de la zona metropolitana de Nuevo León. Como criterio de inclusión se utilizó la mayoría de edad en los participantes y el uso de alguna actividad en su tiempo libre para manejar su estrés. Fueron excluidos menores de edad, cuestionarios donde se declarase no sentirse estresados, el no utilizar alguna actividad para manejar el estrés durante el tiempo libre e instrumentos incompletos.

\section{Instrumento}

Ésta investigación empleó preguntas abiertas para conocer la principal actividad utilizada por los participantes durante su tiempo libre para manejar su estrés, lugar de procedencia y edad. Y dos de opción múltiple para los rubros de sexo y escolaridad.

Como ya se comentó la escala de uso del tiempo libre para el afrontamiento (LCSS; Iwasaki \& Mannell, 2000) es un inventario enfocado al uso de los pasatiempos/tiempo libre como estrategia de afrontamiento ante el estrés, el cual consta de 18 ítems divididos en tres subescalas, las cuales se responden en una escala ordinal de tipo Likert de siete alternativas de respuesta, entre "totalmente de acuerdo" y "totalmente en desacuerdo" con puntuaciones intermedias.

\section{Procedimiento}

Con la finalidad de asegurar la equivalencia conceptual de los ítems originales con su versión en español, se hizo su traducción directa y la revisión de equivalencia (Carretero-Dios \& Pérez, 2005) con apoyo de las definiciones conceptuales aportadas por Iwasaki \& Mannell (2000). La traducción fue realizada por uno de los investigadores y revisada por dos traductores, llegando a una versión de consenso. La versión final de la escala fue aplicada a una muestra piloto de diez individuos con características similares a la de la población objetivo a fin de detectar ítems problemáticos, la claridad de las instrucciones, erratas, el tiempo para 
completar el inventario y la adecuación semántica y sintáctica de los ítems. El instrumento fue administrado como parte de un estudio mayor, en conjunto con las escalas de Estrés Percibido (PSS) de Cohen, Kamarak \& Mermelstein (1983), en su adaptación para México realizada por González y Landero (2007) y la Escala de Satisfacción con la Vida, de Diener, Emmons, Larsen \& Griffin (1985) traducida por Laca, Verdugo \& Guzmán (2005). Las escalas fueron proporcionadas a los participantes a término de una clase dentro de los talleres de un museo, en donde se extendió la invitación de participar voluntaria y anónimamente en el estudio; si bien se trata de un instrumento de autoevaluación para su aplicación se pidió la colaboración de personal del museo, mismo que fue capacitado por una de las investigadoras.

\section{Procedimiento de análisis estadístico}

Para determinar la pertinencia del análisis factorial y la probabilidad de correlaciones significativas entre las variables se aplicó la prueba de esfericidad de Bartlett. Posteriormente se realizó el análisis factorial exploratorio (AFE) con el método de componentes principales y rotación varimax. A fin de evaluar la confiabilidad del instrumento se realizó un análisis de confiabilidad inter-ítem con la prueba Alpha de Cronbach para los ítems que formaron cada subescala. Una vez determinados los factores, se realizó el análisis factorial confirmatorio (AFC) para ratificar el modelo obtenido en el exploratorio. Se empleó el método de máxima verosimilitud para analizar las correlaciones.

\section{Resultados}

De la muestra original $(n=175)$, quince participantes reportaron no realizar ninguna actividad para manejar su estrés, dos reportaron no sentirse estresados y 17 encuestas $(9.7 \%)$ fueron desechadas por estar incompletas; como resultados en éste estudio participaron 141 personas. Respecto a sus características, fueron incluidos $49(34.8 \%)$ varones y $92(65.2 \%)$ mujeres, todos ellos mayores de edad, con una edad promedio de 32.06 años (DT $=9.19$ años) y con un rango de edad entre 18 a 60 años.

\section{Correlación entre los ítems.}

El análisis de fiabilidad de cada subescala se presentan en la Tabla 1; como puede observarse en una de las tres subescalas (afrontamiento paliativo y el tiempo libre/pasatiempo) su valor supera .80 por lo que puede considerarse como "muy buena" (DeVellis, 2003); dos subescalas, "Compañerismo en el tiempo libre/pasatiempo" y "Mejoría del humor mediante el pasatiempo", presentaron un nivel "respetable" de fiabilidad: .776 y .778 respectivamente. La correlación de ítems por subescala se muestran en la Tabla 2.

Tabla 1.

Alpha de Cronbach por subescalas

\begin{tabular}{lcc}
\hline Subescala & $\begin{array}{c}\text { Número de } \\
\text { ítems }\end{array}$ & $\begin{array}{c}\text { Alpha de } \\
\text { Cronbach }\end{array}$ \\
\hline $\begin{array}{l}\text { "Compañerismo en el tiempo libre/ } \\
\text { pasatiempo" }\end{array}$ & 6 & .726 \\
$\begin{array}{l}\text { "Afrontamiento paliativo y el tiempo } \\
\text { libre/pasatiempo" }\end{array}$ & 6 & .835 \\
$\begin{array}{l}\text { "Mejoría del humor mediante el } \\
\text { pasatiempo" }\end{array}$ & 6 & .778 \\
\hline
\end{tabular}

Fuente: elaboración propia.

Con respecto a la validez convergente, los resultados mostraron la correlación significativas entre la Escala de uso del tiempo libre para el afrontamiento y Escala de Satisfacción con la Vida. Una a nivel general, es decir, en los resultados totales de ambas escalas $(r=$ $.207, \mathrm{p}<0.05$ ) y otra entre la subescala "Compañerismo en el tiempo libre/pasatiempo" con la puntuación total de la Escala de Satisfacción con la Vida $(r=$ $.171, \mathrm{p}<0.01)$. Así mismo se obtuvo una correlación significativa negativa entre las escalas de Satisfacción con la Vida y Estrés Percibido $(r=-.363$, $\mathrm{p}<0.01)$ en ésta muestra. La tabla 3 muestra las correlaciones por ítem, entre escalas.

\section{Análisis factorial exploratorio}

El valor correspondiente a la prueba de KaiserMeyer-Olkin (KMO) de la escala fue de .828 y la prueba de esfericidad de Bartlett fue significativa $\left(\mathrm{x}^{2}\right.$ $=1602,519 ; g l=153 ; p<.001)$; los valores obtenidos en el índice KMO y en la prueba de esfericidad de Bartlett por subescala se muestran en la Tabla 4. Debido a que se buscaba confirmar la estructura factorial de la LCSS, se realizó el AF restringiendo a 3 factores. Se confirmó la distribución de ítems en tres dimensiones, al tomar como criterio de inclusión un peso factorial mayor o igual a .40 (Hair et al., 1998). Los ítems se agruparon en tres factores. En el primer factor, se cargaron los ítems de la subescala 
Tabla 2

Correlación inter ítem por subescala y análisis de confiabilidad con ítem eliminado

\begin{tabular}{llcc}
\hline Subescala & Ítems & $\begin{array}{c}\text { Correlación total } \\
\text { inter-ítem }\end{array}$ & $\begin{array}{c}\text { Alpha de Cronbach con } \\
\text { item eliminado }\end{array}$ \\
\hline Compañerismo en el tiempo & Apoyo compañeros & .654 & .776 \\
libre/pasatiempo & Socialización y estrés & .631 & .782 \\
& Pasatiempo compartido & .781 & .751 \\
& Manejo estrés grupo & .667 & .773 \\
& Falta de compañía y estrés & .221 & .868 \\
Afrontamiento paliativo y & Grupo como estrategia & .630 & .782 \\
el tiempo libre/pasatiempo & Pasatiempo como escape & .406 & .857 \\
& Escape y estrés & .693 & .792 \\
& Importancia tiempo libre & .714 & .792 \\
Mejoría del humor mediante & Tiempo libre y perspectiva & .597 & .811 \\
& Escape y energía renovada & .713 & .787 \\
& Tiempo libre como descanso & .605 & .809 \\
& Pasatiempo y sentirse mejor & .782 & .692 \\
& Mejoría ánimo y pasatiempo & .789 & .690 \\
& Buen humor y pasatiempo & .765 & .695 \\
& No mejora humor & .198 & .845
\end{tabular}

Fuente: elaboración propia.

Tabla 3

Correlación por ítems entre las escalas LCSS-M, PSS y SV*.

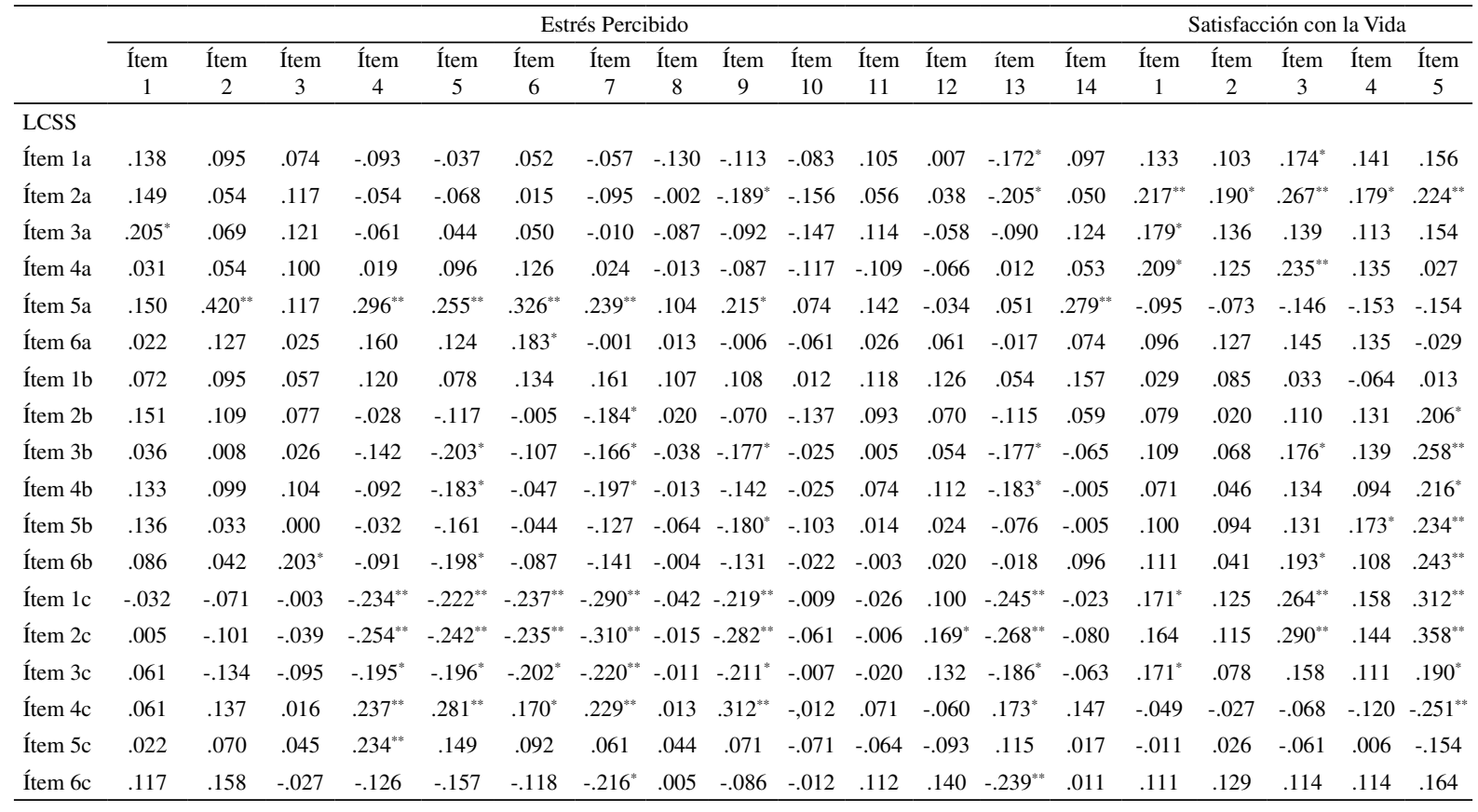

$* p=0.05 * * p=0.01$

Nota: Corresponde LCSS: Escala del uso de tiempo libre para el afrontamiento; PSS: Escala de Estrés Percibido y SV: Escala de Satisfacción con la Vida. 
de "Afrontamiento paliativo y el tiempo libre/pasatiempo"; en el segundo la subescala "Compañerismo en el tiempo libre/pasatiempo" y en tercero "Mejoría del humor mediante el pasatiempo"; lo que confirmó la distribución de ítems en tres subescalas de la
LCSS (Tabla 5). La varianza explicada por los dos factores es de 57.037. La eliminación de la subescala de "Mejoría del humor mediante el pasatiempo" en el AFE se respaldó en el resultado del análisis de confiabilidad.

Tabla 4

Medidas de adecuación muestral Kaiser-Meyer-Olkin y pruebas de esfericidad de Bartlett.

\begin{tabular}{lcccc}
\hline & & \multicolumn{3}{c}{ Prueba de esfericidad de Bartlett } \\
\cline { 3 - 5 } Subescala & KMO & $\mathrm{X}^{2}$ & $\mathrm{gl}$ & $\mathrm{p}$ \\
\hline Compañerismo en el tiempo libre/pasatiempo & .763 & 405.660 & 15 & .000 \\
Afrontamiento paliativo y el tiempo libre/pasatiempo & .812 & 369.035 & 15 & .000 \\
Mejoría del humor mediante el pasatiempo & .842 & 555.553 & 15 & .000 \\
\hline
\end{tabular}

Fuente: elaboración propia.

Tabla 5.

Cargas factoriales de los ítems de la escala LCSS.

\begin{tabular}{lc}
\hline Factor 1 & Carga factorial \\
\hline Pasatiempo como escape & .619 \\
Escape y estrés & .796 \\
Importancia tiempo libre & .763 \\
Tiempo libre y perspectiva & .693 \\
Escape y energía renovada & .770 \\
Tiempo libre como descanso & .726 \\
Factor 2 & \\
Apoyo compañeros & .797 \\
Socialización y estrés & .790 \\
Pasatiempo compartido & .876 \\
Manejo estrés grupo & .809 \\
Falta compañía y estrés & .321 \\
Grupo como estrategia & .718 \\
Factor 3 & \\
Pasatiempo y sentirse mejor & .696 \\
Mejoría ánimo y pasatiempo & .652 \\
Buen humor y pasatiempo & .565 \\
No mejora humor & .422 \\
Pasatiempo y miseria & .743 \\
Pasatiempo y manejo de sentimiento & $.136 *$ \\
negativo & \\
\hline Fuente: elaboracion propia & \\
\hline
\end{tabular}

Fuente: elaboración propia.

Nota: En el caso del último ítem (*) el peso factorial mayor $(, 539)$ se presentó en el primer factor.

\section{Análisis factorial confirmatorio}

Se realizó el análisis factorial confirmatorio de los ítems de la LCSS-M (Figura 1) a fin de ratificar lo obtenido en el exploratorio y determinar las relaciones con el modelo proporcionado por los autores de la escala original, que sostiene la existencia de tres factores; para ello se utilizó el programa de ecuaciones estructurales AMOS 20, en donde se empleó el método de máxima verosimilitud. Como se observa en la figura 1 se estimaron las covarianzas entre algunos errores para mejorar la bondad de ajuste del modelo.

El contraste del ajuste de los datos al modelo teórico se evaluó mediante diversos indicadores de bondad de ajuste (Tabla 6); en concreto se utilizaron la $x^{2}$ divida por los grados de libertad, el error cuadrático medio de aproximación (RMSEA) e indicadores de ajuste de incremento (relativo) como el índice de bondad de ajuste (GFI) y el índice de ajuste comparativo $(\mathrm{CFI})$.

Tabla 6

Índices de bondad de ajuste para el análisis factorial confirmatorio.

\begin{tabular}{lllllllllll}
\hline & & & \multicolumn{3}{c}{$\begin{array}{c}\text { Índices de ajuste } \\
\text { absoluto }\end{array}$} & \multicolumn{3}{c}{$\begin{array}{c}\text { Índices de ajuste de } \\
\text { incremento }\end{array}$} \\
\hline $\mathrm{X}^{2}$ & g.1. & $\mathrm{X}^{2} / \mathrm{gl}$ & GFI & AGFI RMSEA & NFI & TLI & CFI & IFI \\
& & & & & & & & & & \\
258.890 & 124 & 2.088 & .833 & .770 & .088 & .846 & .891 & .912 & .914 \\
\hline
\end{tabular}


Los resultados de los diferentes índices de ajuste utilizados confirman que el modelo propuesto por los autores originales se ajusta a los datos, al estimar las covarianzas entre errores. Puede observarse que los valores de $\mathrm{x}^{2}$ dividida por los grados de libertad presenta un valor cercano al 2, considerado como bueno (Hair et al., 1998) y que error cuadrático de aproximación (RMSEA) se encuentra cercano al ,08 recomendado
(Hooper, Coughlan \& Mullen, 2008); por otro lado puede observarse que el CFI se sitúa por encima del valor 90 recomendado y el índice GFI cercano (Hair et al., 1998). Teniendo en cuenta lo anterior, los datos de la muestra utilizada otorga un apoyo empírico a la estructura triárquica del LCSS, una vez realizado el ajuste.

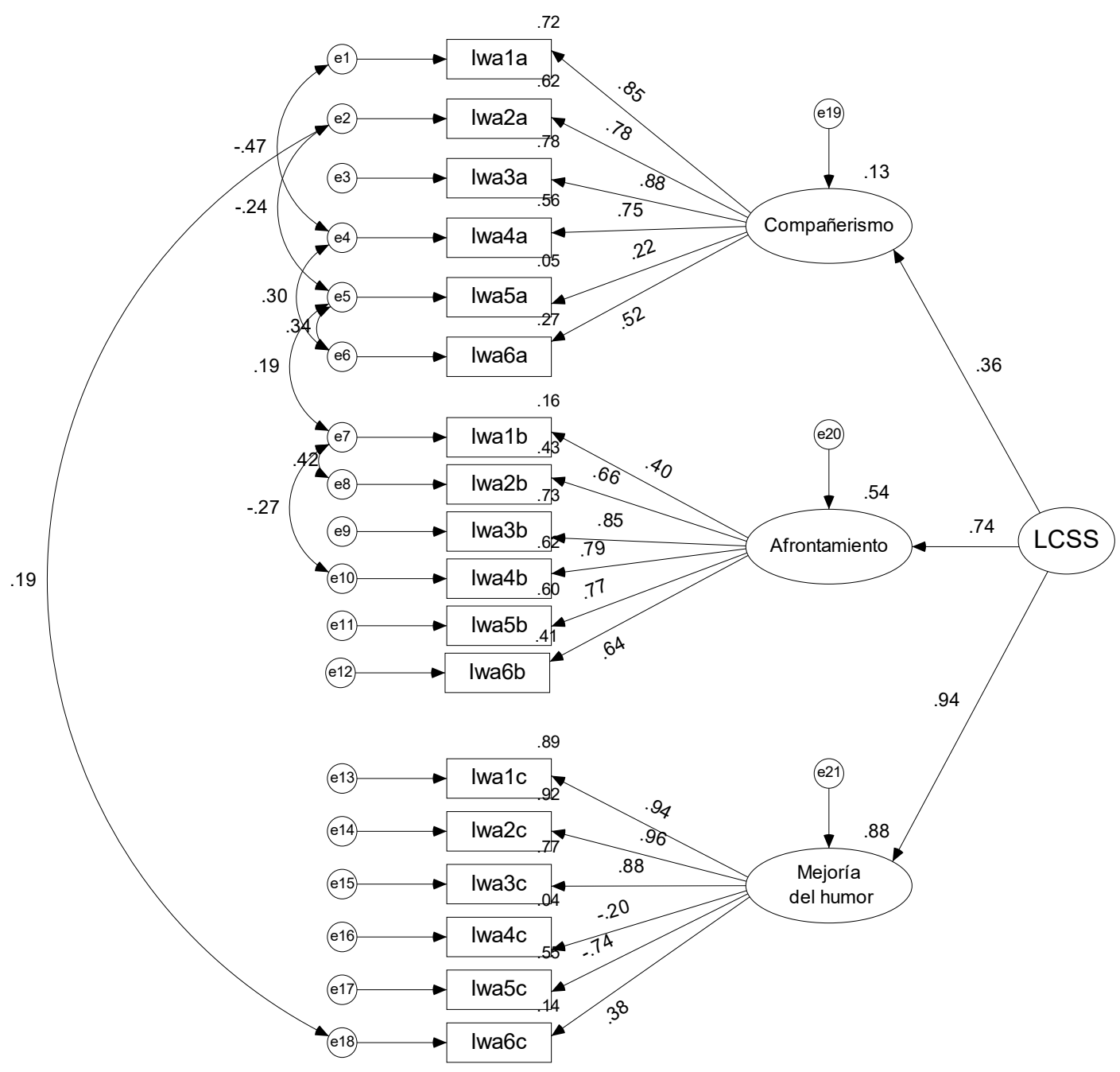

Figura 1

Análisis factorial confirmatorio de los constructos de la LCCS-M. 


\section{Discusión}

Para éste estudio se planteó el objetivo de analizar las propiedades psicométricas de la escala de uso del tiempo libre para el afrontamiento (LCSS; Iwasaki \& Mannell, 2000). Respecto a las propiedades psicométricas de la LCSS-M (Leisure coping strategy scaleMexican version), en el análisis factorial exploratorio el valor de la prueba KMO fue adecuado tanto para la escala general como para cada una de las subescalas, lo que hizo factible el análisis de los ítems que forman la escala de uso del tiempo libre para el afrontamiento (LCSS; Iwasaki \& Mannell, 2000), de ésta manera se confirmó la estructura factorial original y la consistencia interna de las subescalas, en donde se observan índices alfa de fiabilidad adecuados de acuerdo a DeVellis (2003): afrontamiento paliativo y el tiempo libre/pasatiempo (.835), compañerismo en el tiempo libre/pasatiempo (.776) y mejoría del humor mediante el pasatiempo (.778). Sin embargo tanto en éste como en el análisis factorial confirmatorio la LCSS-M muestra debilidades psicométricas relacionadas con la ambigüedad factorial de determinados ítems. Sin embargo, la eliminación de estos ítems no parece mejorar el ajuste del modelo según el análisis factorial confirmatorio; mas sí la estimación de las covarianzas entre ítems.

Es de interés mencionar que, al carecer de otras escalas que evalúen las formas de afrontamiento específicas al área de los pasatiempos y uso del tiempo libre, se dificulta la comparación de los ítems de la escala de uso del tiempo libre para el afrontamiento (LCSS; Iwasaki y Mannell, 2000), por tal motivo fueron utilizadas dos escalas validadas en nuestra población cuyos ítems pudiesen proporcionar datos sobre la validez convergente y discriminante entre ellas. De ésta manera fue posible localizar correlaciones entre la Escala de uso del tiempo libre para el afrontamiento y la escala de Satisfacción con la vida (Diener et al., 1985) y observar la independencia de los constructos abordados mediante la Escala de uso del tiempo libre y los resultados del nivel de estrés percibido; hallazgo que corrobora su relación con el bienestar de las personas pero no de manera inmediata en su estrés (Iwasaki \& Mannell, 2000; Mattingly \& Sayer, 2006). Debido a lo anterior se recomienda la comparación de la escala con otras que contengan ítems relacionados, así mismo el desarrollo de investigaciones enfocadas al uso de pasatiempos y tiempo libre como estrategia de afrontamiento. En resumen, respecto a las propiedades psicométricas del LCSS-M, el análisis de las relaciones entre las variables en el AFC parece apoyar el modelo original definido por Iwasaki \& Mannell (2000); y las propiedades psicométricas de las subescalas que componen el cuestionario muestran índices adecuados por lo que la LCSS-M es una medida válida y fiable para ser empleada en estudios posteriores.

\section{Referencias}

Bueno, J., Fernández-Castro, J. \& Capdevelia, L. (2001). Validez factorial y predictiva del Cuestionario de Afrontamiento en Deportes de Resistencia (CADER): un estudio exploratorio. Ansiedad y Estrés, 7(1), 29-44.

Cano, F., Rodríguez, L. \& García, J. (2007). Adaptación española del Inventario de estrategias de afrontamiento. Acta Española de Psiquiatría, 35(1), 29-39. Recuperado de http://www.researchgate. net/publication/235419673_Adaptacin_Espaola_del_Inventario_de_Estrategias_de_Afrontamiento

Carretero-Dios, H. \& Pérez, C. (2005). Normas para el desarrollo y revisión de estudios instrumentales. International Journal of Clinical and Health Psychology, 5(3), 521-551. Recuperado de http://www.aepc.es/ijchp/articulos_pdf/ijchp-158.pdf

Cohen, S., Kamarck, T. \& Mermelstein, R. (1983). A Global Measure of Perceived Stress. Journal of Health and Social Behavior, 24, 385-396. Recuperado de http://www.psy.cmu.edu/ scohen/ globalmeas83.pdf

DeVellis, R. F. (2003). Scale development. Theory and applications. Thousand Oaks: Sage Publications.

Diener, E., Emmons, R., Larsen, J. \& Griffin, S. (1985). The Satisfaction With Life Scale. Personality Assessment, 49(1), 71-75. doi:10.1207/s15327752jpa4901_13

González, M. \& Landero, R. (2007). Factor structure of the perceived stress scale (PSS) in a sample from Mexico. The Spanish Journal of Psychology, 10(1), 199-206. Recuperado de http://www.psy. cmu.edu/ scohen/Factor\%20Structure_Mexico.pdf

Hair, J., Anderson, R., Tatham, R. \& Black, W. (1998). Multivariate data analysis. Upper Saddle River: Prentice Hall.

Hamarat, E., Thompson, D., Zabrucky, K., Steele, D., Matheny, K. \& Aysan, F. (2001). Perceived stress and coping resource availability as predictors of life satisfaction in young, middle aged, and older adults. Experimental Aging Research, 27, 181-196. doi:10.1080/036107301750074051

Hernández, G., Olmedo, E. \& Ibañez, I. (2003). Estar quemado (burnout) y su relación con el afrontamiento. International Journal of Clinical and Health Psychology, 4(2), 323-336. Recuperado de http://www.aepc.es/ijchp/articulos_pdf/ijchp-110.pdf

Hooper, D., Coughlan, J. \& Mullen, M. (2008). Structural equation modeling: Guidelines for determing model fit. Electronic Journal of Business Research Methods, 6(1), 53-60. Recuperado de http:// www.ssnpstudents.com/wp/wp-content/uploads/2015/02/ejbrmvolume6-issue1-article183.pdf

Iwasaki, Y. \& Mannell, R. (2000). Hierarchical dimensions of leisure stress coping. Leisure Sciences, 22, 163-181. doi: 10.1080/01490409950121843

Laca, F., Verdugo, J. \& Guzmán, J. (2005). Satisfacción con la vida de algunos colectivos mexicanos: una discusión sobre la psicología del bienestar subjetivo. Enseñanza e Investigación en Psicología, 10(2), 325-336. Recuperado de http://www.redalyc.org/articulo. oa? $\mathrm{id}=29210208$

Lazarus, R. \& Folkman, S. (1987). Transactional theory and research on emotions and coping. European Journal of Personality, 1, 141169. doi:10.1002/per.2410010304 
Mactavish, J. \& Iwasaki, Y. (2005). Exploring perspectives of individuals with disabilities on stress-coping. Journal of Rehabilitation, 71(1), 20-31.

Mattingly, M. \& Sayer, L. (2006). Under (pressure): gender differences in the relationship between free time and feeling rushed. Journal of Marriage and Family, 68, 205-221. doi:10.1111/j.17413737.2006.00242.x

Meltzoff, J. (2011). Critical thininking (thinking) about research. Psychology and related fields. Wachington: American Psychological Association.

Miller, T. \& McCool, S. (2003). Coping with stress in outdoor recreational settings: an application of transactional stress theory. Leisure Sciences, 25, 257-275. doi:10.1080/01490400306562

Morán, C., Landero, R. \& González, M. (2010). COPE-28: un análisis psicométrico de la versión en español del Brief COPE. Universitas Psychologica, 9(2), 543-552. Recuperado de http://www.scielo. org.co/pdf/rups/v9n2/v9n2a20

Nava, C., Ollua, P., Vega, C. \& Soria, R. (2010). Inventario de estrategias de afrontamiento: una replicación. Psicología y Salud, 20(2), 213-220. Recuperado de http://www.uv.mx/psicysalud/ psicysalud-20-2/20-2/Carlos-Nava-Quiroz.pdf

Nava-Quiroz, C., Vega-Valero, C. \& Soria, R. (2010). Escala de modos de afrontamiento: consideraciones teóricas y metodológicas. Universitas Psychologica, 9(1), 139-147. Recuperado de http:// www.scielo.org.co/pdf/rups/v9n1/v9n1a11
Olmedo, E., Ibáñez, I. \& Hernández, L. (2001). Validación estructural del Ways of coping questionnaire de Lazarus y Folkman: un análisis factorial restrictivo. Ansiedad y Estrés, 7(1), 15-28.

Ortin, F., Olmedilla, A., Garcés de los Fayos, E. \& Hidalgo, M. (2008). Locus de control y vulnerabilidad a la lesión en fútbol semiprofesional y profesional. Cuadernos de Psicología del Deporte, 8(2), 101-112.

Paris, L. \& Omar, A. (2009). Estrategias de afrontamiento del estrés como potenciadoras de bienestar. Psicología y Salud, 19(2), 167-175. Recuperado de http://www.uv.mx/psicysalud/psicysalud-19-2/19-2/Laura-Paris.pdf

Paz-Rodríguez, F. \& Vázquez-Velez, S. (2012). Afrontamiento y calidad de vida en díadas ante un evento vascular cerebral. XIV Congreso Mexicano de Psicología Social y VI Congreso Mexicano de Relaciones Personales, Investigación presentada en el encuentro de la Asociación Mexicana de Psicología Social y la Facultad de Psicología de la Universidad Autónoma de Nuevo León, Monterrey, México.

Peralta-Ramírez, M., Robles-Ortega, H., Navarrete-Navarrete, N. \& Jiménez-Alonso, J. (2009). Aplicación de la terapia de afrontamiento del estrés en dos poblaciones con alto estrés: pacientes crónicos y personas sanas. Salud Mental, 32(3), 251-258. Recuperado de http://www.redalyc.org/pdf/582/58212279009.pdf 
